of the cokes made from them. As judged by the figures given in Table 3 for the degradation factor and reactivity for both the commercial cokes and those prepared in the experimental oven, it will be seen that (with the exception already noted in Table 3 ) the cokes from coals $A$ and $D$ have lower values than those from coals $B$ and $C$, that is, the former pair of coals give the harder and more unreactive cokes. Moreover, as regards the relative behaviour of the cokes in industrial practice, those made from coals $A$ and $D$ give a very hot and narrow zone in hightemperature shaft furnaces, as compared with a wider and not so hot zone with the cokes from coals $B$ and $C$, as well as a higher temperature in cupolas.

From the results obtained so far in these preliminary experiments, it would appear that by the use of these techniques there is possible a differentiation of coals in respect of at least some of the properties of the cokes produced therefrom. A considerable number of coals are now being investigated to see if there can be found in this way an understanding of the fundamental differences in coals which affect their coking properties.

${ }^{1}$ See Berkowitz, N., Nature, 163, 809 (1949).

'Banerjee, S., and Sarjant, R. J., F'uel, 30, 130 (1951)

Bone, W. A., and Sarjant, R. J., Proc. Roy. Soc., A, 96, 119 (1919). "For example, "Infra-Red Determination of Organic Structures", by Randall, H. M., lowler, R. (K., Fuson, N., and Dangl, J. R.' (D Van Nostrand Co., Inc., New York, 1949). Cannon, (. G., and land, G. B. B. M., Discuss. Farad. Soc., No. 9, 274 (1950).

\section{FIRST INTERNATIONAL CONGRESS ON ANALYTICAL CHEMISTRY, OXFORD}

$\mathrm{T}$

HE First International Congress on Analytical Chemistry, under the patronage of the international Union of Pure and Applied Chemistry, was held at Oxford during September 4-9, with Sir Robert Robinson as president. The Congress consisted of twelve sessions, at which forty-three scientific papers, under nine subject groups, were read and discussed, and of four Congress lectures. There were also a trade exhibition of instruments, apparatus and books, and an exhibition of analytical techniques and apparatus.

The success of the meeting was assured from the start by the attendance of about seven hundred chemists from all over the world, some of whom were also able to attend meetings of the Section Committee and Commissions of the Analytical Chemistry Section of the International Union before and after the Congress. The success of the Congress was a worthy tribute to the organizers, the burden of the work falling on Mr. R. C. Chirnside, whose ability was reflected in the smooth running of all the proceedings.

The first of the Congress lectures was given by Prof. I. M. Kolthoff, of the University of Mimnesota, who spoke on the "Ageing of Crystalline l'recipitates". The 'ageing' to which Prof. Kolthoff referred denotes the irreversible structural changes that occur in a precipitate after it has been formed, of which the classical 'Ostwald ripening', the growth of larger particles at the expense of the smaller, is an example.

Dr. R. H. Müller, of Los Alamos Laboratory, New Mexico, an authority on the subject of instrumentation, spoke on "Research in Analytical Instru- mentation". His lecture was not a mere summary but reviewed the possibilities for the future. He discussed the need for perfected instruments such as high-speed, auto-recording balances and instruments for recording refractive index and for photoelectric titration.

Dr. L. H. Lampitt, chief chemist of J. Lyons and Co., Ltd., Laboratories, London, gave the third Congress lecture, with the title "The Value and Economic Importance of Chemical Analysis in Industry and Manufacture". He traced developments from the chemical revolution of the seventeenth century to the present time, and pointed out that to-day the analyst is in a position to aid industry in the main fields of commercial activities, namely, research, process development and process control. He stressed how, in industry, analytical control could, by means of instrumentation, be carried out by relatively unqualified (in the academic sense) persons, and their work could be assessed as a 'benchrate', a prime charge against the article manufactured.

Finally, Prof. van Nieuwenburg, professor of chemistry in the T'echnical University of Welft, spoke on "A Contemporary Assessment of the Place of Classical Methods in Chemical Analysis". He spoke of the revolutionary changes in chemical analysis and of the fact that most of the papers read at the Congress were on matters or techniques unheard of twenty-five years ago. The classical methods, which are essentially chemical, may be losing ground, but they still have a future, as the new physical methods need them as a basis.

The papers read at the Congress and the discussions that followed are to be published in the November and December numbers of The Analyst, and the whole of the proceedings will be available as a separate bound volume for members of the Congress and others. Consequently, the papers are not here reviewed in detail, but a brief survey is given of the general and specialized subjects discussed.

Five papers on microchemical methods were read, that on the direct micro-determination of oxygen in organic substances being particularly interesting. The apparatus involved was shown at the apparatus exhibition. The micro-gravimetric determination of lead as sulphate and of silver and of mercurous mercury as chlorides was described; the determination of fluorine by the lead chlorofluoride method in compounds containing halogens, sulphur, phosphorus and arsenic was reviewed; and other papers included a description of a countercurrent micro-rotary extractor and a summary of some of the approaches that have been utilized in the development of spot tests, using either organic or inorganic reagents.

In the subject group of optical methods of analysis, there were six papers, covering two sessions. These papers served to illustrate the greater value of 'open' subjects when they are intended for general discussion at meetings. For example, an account of recent advances in infra-red spectroscopic techniques and a paper on the colorimetry of indicators both aroused keen discussion. On the other hand, the papers on ultra-violet absorptiometric determination of arsenic as 12-molybdoarsenic acid (designed for large percentages of arsenic) and on ultra-violet absorption spectra of pyridoxine and related compounds were only of interest to a limited number of people, although valuable in print. In addition, the application of emission spectrography to the routine determination of metals soluble in lubricating oils 
and the identification by X-ray diffraction of crystalline inclusions in glass were discussed.

The session on the presentation of data moved briskly under a forceful chairmanship. Two papers which were taken together and which aroused considerable interest were those on statistical aspects of analytical determinations, for the detection and correction of sources of error in analytical procedures, and on statistical design in the study of analytical methods, to determine the sensitivity of an analytical method. The third paper in this subject group was on statistical methods in radiochemistry.

The morning of September 6 was devoted entirely to the study of radiochemical methods, and this naturally attracted the chemists working in the newer fields of atomic research. A paper on the determination of trace quantities of the alkali metals by neutron activation analysis claimed that this type of analysis is unique because of its great sensitivity and the fact that other elements do not interfere. Two papers were read on the determination of small quantities of uranium by radioactivation and on precision counting of $\alpha$-particles, while the preparation of astatine by bombardment of bismuth oxide with helium ions and the solvent extraction of Group III $B$ metal halides with the aid of radionuclides were also discussed.

The session on organic complexes opened with a paper on some factors controlling the selectivity of organic reagents, in which a product $M L_{n}$, formed from a cation $M$ and an acidic reagent $\mathrm{H} L$, if it has suitable physical properties, may serve as a basis for a test for $M$. The stability of metal 'chelates' in relation to their use in analysis, and 1:10-phenanthroline and mono-, di-, tri- and tetra-methyl1 : 10-phenanthrolines as chelated copper complex cations were studied; also discussions took place on the complexes formed by thorium and uranyl ions with complexones (that is, derivatives of iminodiacetic acid) and on the fractionation of ozokerites and the separation of optical isomers.

A general survey on a fundamental approach to the establishment of $p \mathrm{H}$ standards opened the session on electrical methods. It was emphasized that $p \mathrm{H}$ can never possess the absolute significance of a true thermodynamic constant. A method of determining the equivalence point in potentiometric titrations, applicable to instruments calibrated either in millivolts or $p \mathrm{H}$ units, was said to have been achiever by a numerical manipulation transforming potentialvolume curves into straight lines intersecting at the equivalence point. Also included were papers on the replacement of the standard cell and salt bridge by indicator eloctrodes and the use of non-aqueous solutions in potentiometry, on square-wave polarography, and on polarography of the tetrathionate ion.

It was perhaps significant that nine papers were presented to the adsorption and partition methods group, involving three separate sessions of the Congress. Recent work on gas chromatography was illustrated by the separation and analysis of volatile organic mixtures by displacement chromatography from charcoal columns at $100^{\circ} \mathrm{C}$. to an accuracy of better than 1 per cent. It was also suggested that some of the limitations of current chromatographic procedures are overcome by gradient elution analysis, although it has certain disadvantages. The theory of the partition column has been extended to include a compressible mobile phase of a permanent gas, and a method of determining the height equivalent to a theoretical plate of such a partition column has beer. given. The apparatus involved was demonstrated at the apparatus exhibition.

The theoretical aspects of paper chromatography have been studied with the aid of semi-automatic instruments based on the square-law behaviour of the ascent or descent of liquid in a strip or sheet of paper. In the field of quantitative inorganic paper chromatography, working details were given for a new chromatographic separation of aluminium, titanium and iron in three individual spots within a time of $330 \mathrm{~min}$. and an accuracy of \pm 2 per cent. The separation of individual groups of water-soluble extracts of plant and animal tissues was achieved by displacement chromatography on ion-exchange resins, a technique that led to the chemical characterization of new or unexpected components.

Other papers included one on the use of electrokinetic ultrafiltration to enable the larger molecules of the polysaccharides to enter the porous materials used for chromatography, the inability to do so being normally a serious difficulty, one paper on the applications of ion exchange to analytical chemistry, and one on the quantitative determination of tantalum and niobium by inorganic chromatography on cellulose. This last technique was demonstrated at the apparatus exhibition.

The less-exact science of biological methods attracted more limited audiences. Papers in this subject group included those on the use of protozoa in analysis; the use of fungi for determining trace metals in biological materials, whereby increased growth in test fungi was stimulated by the addition of graded amounts of certain metals, from deficiency to sufficiency levels, to a culture solution containing all the other essential nutrients; and the quantitative assay of pyrogens by the febrile response in rabbits, using selected animals according to their body temperature, body weight, sensitivity, excitation and tolerance.

In the session devoted to general subjects a titrimetric method was described for the rapid analysis of certain binary liquid mixtures to which alternative methods may not be readily applicable, and the quantitative determination of organic halides by evaluation of reaction velocity curves was discussed.

The exhibition of analytical techniques and apparatus, so efficiently organized by Mr. F. R. Jones, was regarded by some as the most important feature of the Congress. Sir Robert Robinson, in expressing the wish that the exhibits could be retained for his students to see, echoed the thoughts of many visitors who felt that it was a minor tragedy to have to dismantle these outstanding exhibits, involving as they did a wide range of subjects and craftsmanship and ingenuity of design of a high order. The usefulness of the exhibition was enhanced by the fact that it was staffed at all hours by an enthusiastic team of demonstrators who were qualified scientific workers, specialized in their particular subjects and always ready to answer questions or to discuss the fundamentals of their exhibits.

It must be clearly understood that selection of the items for description in this article has been quite arbitrary and that only lack of space precludes the inclusion of them all. Some, it is understood, have been, or are to be, published in the Journal of Scientific Instruments.

In the case of three of the scientific papers described above, reference was made to the fact that the instruments or techniques involved were also demon- 
strated at the exhibition. These included: an apparatus for the determination of oxygen in organic compounds, in which the products of pyrolysis of the compound are passed in a stream of oxygen-free nitrogen over carbon at $1,120^{\circ}$ C., the carbon monoxide produced being oxidized by iodine pentoxide at $120^{\circ} \mathrm{C}$. and the liberated iodine oxidized to iodate and determined by thiosulphate; the use of partition chromatography involving a mobile gas phase and a stationary liquid phase to separate and estimate micro-amounts of volatile fatty acids from formic acid to lauric acid, including all the isomers of valeric acid; and the application of paper strip technique, widely used in organic separations, to inorganic materials covering a very wide range, and the application of the method to separations on a larger scale using columns of cellulose powder.

A popular exhibit was that demonstrating remotehandling devices for radioactive work. It comprised a 'gamma handling cell' for the safe handling of $100 \mathrm{mC}$. of $1-\mathrm{MeV}$. gamma-ray sources, tongs for changing tong-heads remotely and a mechanical simulator which is a model of a machine used for accurate manipulation from behind a dense shieldingwall-for example, 2-3 ft. of concrete. An excellent instrument was shown, known as the 'thermistor ebullioscope'. This instrument is intended for the rapid and precise routine determinations of molecular weight. Rapidity is achieved by employing thermistors as short time-constant differential thermometers, and precision by the use of specially designed vapourjacketed ebullioscopes. Boiling-point elevations, accurate to $0.0005 \mathrm{deg}$. or less, can be read within about two minutes of adding the solute.

A large and beautifully laid-out exhibition of microchemical apparatus had been organized by the Norwood Technical College (London County Council). This exhibit aroused considerable interest, and the demonstrators were tireless in answering questions: Most of the items were of very simple construction, but the success of the exhibit lay in the fact that the large number of pieces of micro-glassware-methods of stirring in small-scale work, micro-conductometric and other electrochemical techniques and apparatus for small-scale organic syntheses and identification and for general microchemistry - had been made and assembled by a team of enthusiasts working in their spare time.

A fascinating demonstration of new methods of microscopy was organized by Dr. R. Barer, of the Department of Anatomy, Oxford. The subject was, of course, highly specialized, but was of considerable interest to all the visitors who saw the demonstration. There were also demonstrations of a new method of identifying crystalline substances by measurement of interfacial angles, and of the Federov universal stage for determining the optical constants of crystals.

Altogether there were eighty-seven exhibits provided by forty-one individuals, firms, laboratories, research associations or colleges. There was also a trade exhibition of instruments, apparatus and books, effectively organized by Mr. W. Thompson, in which forty-four trade exhibitors were represented.

Apart from the purely scientific side of the Congress, the social side was catered for in a simple but effective manner by the arrangement of tours and excursions. Several informal receptions took place, and a banquet was held in the Hall of Christ Church. The Congress was undoubtedly a great success, and the next one will be awaited with pleasurable anticipation.

\section{ELECTRICAL DISCHARGES IN GASES}

$A^{T}$ T the recent Belfast meeting of the British Association, a session was held on Septembe 8 by Section A (Mathematics and Physics) which was devoted to electrical discharges in gases. The rather full programme included six papers on important aspects of this subject and a discussion. Prof. A. M. Tyndall presided.

The application of the subject of electrical discharges to-day covers a wide range in physics, including, for example, the investigation of certain properties of matter; the nature of ion-atom reactions; metal surface phenomena in discharge tubes, Geiger counters, and electrical contact devices of every kind; radar switches and the generation of radio-frequency oscillations ; high-voltage insulation by compressed gases; and ion sources for nuclear physics. The strong interest was reflected in the full attendance, which included a number of electrical engineers.

The session was opened with a paper by Prof. F. Llewellyn Jones (University College, Swansea) on the electrical breakdown of gases. Discharge phenomena at low pressures, at atmospheric pressures, in highly compressed gases and at electrical contacts appear very different, and different theories have been proposed. Recent work, however, suggests that there are basic principles common to all these aspects. Breakdown at low pressures has been shown to be controlled by primary ionization with a secondary process determined by the nature and geometry of the cathode. For sparks at atmospheric pressure an entirely different theory depending on space-charge effects has been proposed, but recent precision measurements of the ionization currents just prior to sparking show that breakdown in uniform fields at this pressure also is controlled by primary and secondary ionization processes the relative importance of which is controlled by the nature of the cathode ${ }^{1}$. No space-charge effects have been found. With high voltages and compressed gases breakdown appears to be different again in that Paschen's law fails, but this can be explained by the additional generation of electrons by field emission from the cathode. Similar effects can occur at electrical contacts ${ }^{2}$. On the generalized theory proposed, breakdown is a consequence of the development of ionization currents in the gas by primary and secondary processes. No single secondary process always predominates; several can and do occur, sometimes together, sometimes singly. The factors which mainly determine the predominant process in any particular case of breakdown are the nature and geometry of the cathode. In this respect, therefore, the physics of electrical breakdown of gases has much in common with the physics of surfaces.

Dr. C. Grey Morgan (University College, Swansea) described an investigation of cold-electron emission both from electrodes covered with thick oxide layers and from those with thin tarnish films. With heavily oxidized metals, of the type often encountered in heavy electrical engineering, emission of $10^{5}$ electrons/sec. was obtained with low electric fields of about $10^{5} \mathrm{~V} . / \mathrm{cm}$. The fundamental physical process underlying this emission is considered to be the extraction of electrons from ionic sites on the thick contaminating oxide layers always present on surfaces operated in the atmosphere ${ }^{1}$. Another class of 